\title{
AUTODUAL CONNECTION IN THE FOURIER TRANSFORM OF A HIGGS BUNDLE*
}

\author{
JUHANI BONSDORFF ${ }^{\dagger}$
}

\begin{abstract}
The Fourier transform of a stable Higgs bundle of degree zero and rank $\geq 2$ on a curve of genus $\geq 2$ is a locally free sheaf on the cotangent bundle $T^{*} \mathrm{~J}(X)$ of the Jacobian of the curve. The base $T^{*} \mathrm{~J}(X)$ can be identified with the fine moduli space of Higgs bundles of rank 1 and degree 0 on $X$ and carries a natural hyper-Kähler structure.

We use a combination of generalised $\mathcal{D}$-module theory and twistor methods to show that the Fourier transform admits a natural connection which is autodual with respect to the hyper-Kähler structure. The construction exploits Deligne's and Simpson's description of the twistor space of the base in terms moduli spaces of $\lambda$-connections.
\end{abstract}

Key words. Higgs bundle, Fourier transform, Nahm transform, autodual connection, hyperholomorphic, hyper-Kahler, twistor space, D-module.

AMS subject classifications. 14H60, 53C07; Secondary 14F10, 32C 38, 53C26, 53C28

1. Introduction. Let $X$ be a smooth complete algebraic curve over an algebraically closed field $k$ (i.e., a compact Riemann surface in case $k=\mathbf{C}$ ), and let $\mathrm{E}=(\mathcal{E}, \theta)$ be a stable Higgs bundle of degree zero on $X$. In [4] we introduced a Fourier-Mukai transform $\widehat{E}$ of $E$, which is an algebraic vector bundle on the cotangent bundle $T^{*} \mathrm{~J}(X)$ of the Jacobian of $X$, and proved that it extends to an algebraic vector bundle on projectivisation of the base.

Following a suggestion of N.J. Hitchin, one expects that in the case where $k=$ C this Fourier transform should carry a natural connection, thus completing the construction of an essentially Nahm-type transformation for Higgs bundles on curves. Taking cue from Jardim [15], one expects that the connection should be autodual in a suitable sense, corresponding to the doubly-periodic instantons in the genus- 1 case. This paper confirms these expectations and completes the construction of the Nahmtype transformation announced in [4] by exhibiting a construction of a connection in the Fourier transform with the expected properties. The results appearing here have been originally proved in the author's thesis [3].

The base manifold $T^{*} \mathrm{~J}(X)$ of the Fourier transform $\widehat{\mathrm{E}}$ can be identified with the fine moduli space $\mathbf{M}_{D o l}(X, 1)$ of Higgs bundles of rank 1 and degree 0 on $X$. By Hitchin [12] it carries a natural hyper-Kähler structure: it is Kähler with respect to three complex structures $I, J$ and $K$ satisfying $I J K=-1$, with $I$ being the complex structure of the Higgs bundle moduli space and $J, K$ being associated to the same underlying set as the moduli space of flat line bundles on $X$.

We show that $\widehat{E}$ admits a natural connection $\nabla$, which is autodual with respect to this natural hyper-Kähler structure: its curvature is invariant under the action of the group $\mathbf{S U}(2)$ of unit quaternions, specified by the actions of the three complex structures $I, J$ and $K$ on the tangent bundle. Equivalently, the connection is hyperholomorphic, or has curvature of type $(1,1)$ with respect to all the complex structures in the hyper-Kähler family of the base (Kaledin-Verbitsky [16]).

Using a result of Kaledin and Verbitsky [16] (generalising one from CapriaSalamon [6]), we reduce the construction of the autodual connection to the construc-

*Received July 11, 2009; accepted for publication January 25, 2010.

†Pietarinkatu 3, 00140 Helsinki, Finland (juhani.bonsdorff@eqt.fi). 
tion of a suitable holomorphic vector bundle on the twistor space of the base. More precisely, the twistor transform gives a bijective correspondence between bundles with autodual connections on a hyper-Kähler manifold $M$ and those holomorphic bundles on the twistor space of $M$ that are trivial along the "horizontal" twistor lines.

The ideas of Deligne [8] (see Simpson [26]) give a description of the twistor space of $\mathbf{M}_{D o l}(X, 1)$ in terms of moduli spaces of $\lambda$-connections, objects that interpolate between Higgs bundles and flat bundles. More precisely, the twistor space can be obtained by glueing the moduli space $\mathbf{M}_{H o d}(X, 1)$ of rank- 1 degree- $0 \lambda$-connections to its own complex conjugate via a canonical antiholomorphic involution. This reduces the construction of the autodual connection to the construction of a locally free sheaf extending the Fourier transform $\widehat{\mathrm{E}}$ to the larger moduli space $\mathbf{M}_{H o d}(X, 1) \supset \mathbf{M}_{D o l}(X, 1)$ in such a way that this extension can be glued to its conjugate to produce a holomorphic bundle trivial along the horizontal twistor lines.

The most convenient set-up for working with $\lambda$-connections and their cohomology is provided by Simpson rings (called split almost-polynomial sheaves of operators in Simpson [24]), a generalisation of the ring $\mathcal{D}_{X}$ of holomorphic linear differential operators. We show that one can extend the usual homological formalism of $\mathcal{D}_{X}$-modules, including de Rham functors, to modules over Simpson rings. The description of the base of the Fourier transform as the fine moduli space of Higgs bundles then allows us to describe $\widehat{\mathrm{E}}$ on $M=\mathbf{M}_{D o l}(X, 1)$ as the first de Rham sheaf $\mathbf{R}^{1} \operatorname{pr}_{M *}\left(\mathbf{D} \mathbf{R}_{X \times M / M}\left(\operatorname{pr}_{X}^{*} \mathrm{E} \otimes \mathcal{L}\right)\right)$ of $\mathrm{E}$ tensored with the universal rank-1 Higgs bundle.

The moduli space $\mathbf{M}_{H o d}(X, 1)$ is also fine, which provides us with a universal family $\mathcal{U}$ of rank- $1 \lambda$-connections extending the universal Higgs bundle. On the other hand, the analytical results of Hitchin [12], Donaldson [9] Corlette [7] and Simpson $[22,23]$ associate to $\widehat{E}$ a natural family $E^{\prime}$ of $\lambda$-connections. The first de Rham sheaf of $E^{\prime}$ tensored with $\mathcal{U}$ now provides the required extension of $\widehat{E}$ to a locally free sheaf $\mathcal{F}$ over the moduli space $\mathbf{M}_{\text {Hod }}(X, 1)$. The usual cohomological formalism extended to Simpson rings guarantees that the fibre-wise cohomology spaces combine into a holomorphic (indeed algebraic) vector bundle over the moduli space.

This approach to constructing the auto-dual connection has the advantage of being completely geometric; indeed, with the exception of the antiholomorphic involution in the construction of the twistor space, our constructions stay within the holomorphic category. The analytical input needed to prove the main result is concentrated in the use of the Hitchin-Donaldson-Corlette-Simpson result in the construction of the "horizontal" family $E^{\prime}$ of $\lambda$-connections and proof of the triviality of $\mathcal{F}$ along the horizontal twistor lines.

In this paper, the next section develops the cohomology of Simpson modules and reviews and complements the theory of $\lambda$-connections and their moduli spaces. The following Section 3 reviews the hyper-Kähler structure of the moduli space of Higgs bundles as well as Deligne's description of its twistor space. Finally, in the last section we review the twistorial characterisation of autodual connections and carry out the construction of the twistor transform of the connection in the Fourier transform.

Acknowledgements. The idea of constructing a type of autodual connection in the Fourier transform of a Higgs bundle is due to Nigel Hitchin. I would like to thank him for a number of very useful discussions. 
Notation and conventions. All schemes are of finite type and separated over C. All morphisms are $\mathbf{C}$-morphisms and all products are products over $\operatorname{Spec}(\mathbf{C})$ unless stated otherwise. A curve always means a smooth irreducible complete (i.e., projective) curve over $\mathbf{C}$, i.e., a compact Riemann surface. We move freely between a scheme $X$ and the associated analytical space $X^{\text {an }}$, and typically denote both by $X$. When the spaces are non-singular we freely make implicit use of the underlying smooth manifold. All schemes occuring in the construction of the autodual connection in Section 4 are non-singular (i.e., smooth over $\mathbf{C}$ ), and a reader that so wishes can safely limit attention to non-singular schemes throughout the paper.

$\mathbf{G}_{m}$ denotes the multiplicative group $\operatorname{scheme} \operatorname{Spec}\left(\mathbf{Z}\left[T, T^{-1}\right]\right.$ ) (or in our context $\left.\operatorname{Spec}\left(\mathbf{C}\left[T, T^{-1}\right]\right)\right)$. It acts naturally on the affine line $\mathbf{A}^{1}$ by multiplication. For a scheme $S$ recall that $\mathbf{G}_{m}(S)$ denotes the points of $\mathbf{G}_{m}$ with values in $S$, i.e., $\operatorname{Hom}\left(S, \mathbf{G}_{m}\right)$. Thus in particular $\mathbf{G}_{m}(\mathbf{C})=\mathbf{C}^{*}$. We denote canonical isomorphisms by "=".

2. Simpson modules and $\lambda$-connections. In this section we set up technical tools that will be applied to study the Fourier transformation. Cohomology of Simpson modules, generalising standard $\mathcal{D}$-module methods, provides a uniform language for treating the cohomology of Higgs bundles and flat bundles varying in universal families. Such families needed in our construction will be parametrised by moduli spaces of $\lambda$-connections that interpolate between flat and Higgs bundles.

2.1. Cohomology of Simpson modules. We recall and complement the theory of generalised $\mathcal{D}_{X}$-modules, introduced in [24] as modules over split almost-complex sheaves of operators, showing in particular that the usual cohomological machinery including de Rham functors works in this context. We shall state and prove the few results that we need in slightly more general form than needed in the sequel as we expect that there should be applications beyond $\lambda$-connections.

In this subsection we let $S$ be a scheme over $\mathbf{C}$ and we fix a smooth $S$-scheme $f: X \rightarrow S$.

Definition 2.1.1. An $\mathcal{O}_{X}$-algebra $\mathcal{A}$ equipped with an exhaustive increasing filtration $\mathcal{A}^{(0)} \subset \mathcal{A}^{(1)} \subset \ldots$ is a Simpson ring on $X$ over $S$ if it satisfies the following conditions:

S1. The $\mathcal{O}_{X}$-module $\mathcal{A}^{(0)}$ is equal to $\mathcal{O}_{X}$,

S2. The pull-back $f^{-1} \mathcal{O}_{S}$ is contained in the centre of $\mathcal{A}$,

S3. The associated graded $\mathcal{O}_{X}$-algebra $\operatorname{gr}^{\bullet} \mathcal{A}$ is isomorphic to $\operatorname{Sym}^{\bullet} \mathcal{T}$ for a locally free $\mathcal{O}_{X}$-module $\mathcal{T}$, and

S4. The projection $\mathcal{A}^{(1)} \rightarrow \operatorname{gr}^{1} \mathcal{A}$ has an $\mathcal{O}_{X}$-linear section $\sigma: \operatorname{gr}^{1} \mathcal{A} \rightarrow \mathcal{A}^{(1)}$ for the left $\mathcal{O}_{X}$-module structure of $\mathcal{A}$.

A left (resp. right) Simpson module shall mean an $\mathcal{O}_{X}$-coherent left (resp. right) module over a Simpson ring $\mathcal{A}$.

2.1.2. The structure of an $\mathcal{A}$-module is fixed already by the action of $\mathcal{A}^{(1)}$ since by $(\mathbf{S} 4) \mathcal{A}$ is generated as a ring by $\mathcal{A}^{(1)}$. It follows that to give a coherent $\mathcal{O}_{X}$-module $\mathcal{M}$ an $\mathcal{A}$-module structure it is sufficient to give the action of $\sigma\left(\mathrm{gr}^{1} \mathcal{A}\right)$ on $\mathcal{M}$. This action has to satisfy certain commutation relations depending on $\mathcal{A}$, see Simpson [24] Lemma 2.13. 
EXAMPLES 2.1.3.

1. The sheaf $\mathcal{D}_{X / S}$ of relative linear differential operators on $X$ is the canonical example of a Simpson ring: $\mathcal{D}_{X / S}^{(k)}$ is the subsheaf of operators of order $\leq k$, and the associated graded is $\operatorname{Sym}^{\bullet} \mathcal{T}_{X / S}$, the symmetric algebra of the relative tangent sheaf. An $\mathcal{O}_{X}$-coherent $\mathcal{D}_{X / S}$-module is precisely a locally free sheaf equipped with a flat connection relative to $S$, and the action of $\sigma\left(\mathcal{T}_{X / S}\right)$ is simply the covariant derivative in the direction specified by a vector field. See Björk [2], Borel [5] or Mebkhout [19] for details about $\mathcal{D}$-modules.

2. The $\mathcal{O}_{X}$-algebra $\mathcal{A}=\operatorname{Sym}^{\bullet} \mathcal{T}_{X / S}=\operatorname{gr}^{\bullet} \mathcal{D}_{X / S}$ is a Simpson ring. An $\mathcal{A}$-module structure on a coherent sheaf $\mathcal{F}$ is an $\mathcal{O}_{X}$-linear morphism $\theta: \mathcal{F} \rightarrow \mathcal{F} \otimes_{\mathcal{O}_{X}} \Omega_{X}^{1}$ which satisfies $[\theta, \theta]=0$. In other words, a left $\mathcal{A}$-module is the same thing as a Higgs sheaf; see Simpson [24] p. 86 for details.

3. Let $D \subset X$ be a divisor with relative normal crossings. Then there is a Simpson ring $\mathcal{D}_{X / S}(\log D)$, with $\operatorname{gr}^{1} \mathcal{D}_{X / S}(\log D)$ equal to the dual of the sheaf $\Omega_{X / S}^{1}(\log D)$ of logarithmic differentials, such that a $\mathcal{D}_{X / S}(\log D)$-module is the same thing as a sheaf $\mathcal{E}$ with a logarithmic connection

$$
\nabla: \mathcal{E} \rightarrow \Omega_{X / S}(\log D) \otimes_{\mathcal{O}_{X}} \mathcal{E}
$$

relative to $S$.

Remark 2.1.4. If $\mathcal{A}$ is a Simpson ring on $X / S$, then $\mathcal{O}_{X}$ has a canonical $\mathcal{A}$ module structure with a section $t$ of $\mathcal{T}=\operatorname{gr}^{1} \mathcal{A}$ acting as the commutator $[\sigma(t), \bullet]$ on $\mathcal{A}^{(0)}=\mathcal{O}_{X}$. For $\mathcal{A}=\mathcal{D}_{X / S}$ this gives the canonical relative flat connection $d_{X / S}: \mathcal{O}_{X} \rightarrow \Omega_{X / S}^{1}$, and for $\mathcal{A}=\operatorname{Sym}^{\bullet} \mathcal{T}_{X / S}$, one has the trivial Higgs bundle $\mathcal{O}_{X}$ with $\theta=0$.

2.1.5. Let $\mathcal{A}$ be a Simpson ring on $X$ over $S$. We denote by $\mathbf{D}^{b}(\mathcal{A})$ the bounded derived category of left $\mathcal{A}$-modules, not necessarily $\mathcal{O}_{X}$-coherent. The subcategory of objects with $\mathcal{O}_{X}$-quasi-coherent cohomology is denoted by $\mathbf{D}_{q c}^{b}(\mathcal{A})$. The standard arguments guarantee the existence of enough injectives and hence right-derived functors.

2.1.6. Let $\mathcal{M}$ and $\mathcal{N}$ be $\mathcal{A}$-modules. We give the tensor product $\mathcal{M} \otimes_{\mathcal{O}_{X}} \mathcal{N}$ a structure of an $\mathcal{A}$-module by letting the action of a section $t$ of $\mathcal{T}$ be

$$
t(m \otimes n)=t m \otimes n+m \otimes t n .
$$

There are enough $\mathcal{A}$-flat modules, as can be seen by the same argument that applies to $\mathcal{D}$-modules (see Borel [5], VI.2.4). It follows from (S3) that $\mathcal{A}$ is $\mathcal{O}_{X}$-flat, and thus any $\mathcal{A}$-flat resolution is also $\mathcal{O}_{X}$-flat. Hence we have the left derived bifunctor

$$
(\bullet) \stackrel{\mathbf{L}}{\otimes_{\mathcal{O}_{X}}}(\bullet): \mathbf{D}^{b}(\mathcal{A}) \times \mathbf{D}^{b}(\mathcal{A}) \rightarrow \mathbf{D}^{b}(\mathcal{A})
$$

of tensor product over $\mathcal{O}_{X}$. The underlying $\mathcal{O}_{X}$-modules of $H^{-p}(\mathcal{M} \otimes \stackrel{\mathbf{L}}{\otimes})$ are the ordinary tor-sheaves $\mathcal{T}_{\text {or }}^{p}{ }_{\mathcal{O}_{X}}^{p}(\mathcal{M}, \mathcal{N})$ of the underlying $\mathcal{O}_{X}$-modules. It follows that ${\stackrel{\mathbf{L}}{\mathcal{O}_{X}}}$ maps $\mathbf{D}_{q c}^{b}(\mathcal{A}) \times \mathbf{D}_{q c}^{b}(\mathcal{A})$ to $\mathbf{D}_{q c}^{b}(\mathcal{A})$. Furthermore, if $\mathcal{M}$ or $\mathcal{N}$ is locally free over $\mathcal{O}_{X}$, all the higher tors vanish.

Next we show that Spenser resolutions of $\mathcal{D}$-modules generalise to Simpson modules. 
2.1.7. Let $\mathcal{A}$ be a Simpson algebra with $\operatorname{gr}^{1} \mathcal{A}=\mathcal{T}$ of rank $n$, and consider the augmented complex of Simpson modules

$$
0 \rightarrow \mathcal{A} \otimes_{\mathcal{O}_{X}} \bigwedge^{n} \mathcal{T} \stackrel{\delta}{\rightarrow} \mathcal{A} \otimes_{\mathcal{O}_{X}} \bigwedge^{n-1} \mathcal{T} \rightarrow \cdots \rightarrow \mathcal{A} \stackrel{\epsilon}{\rightarrow} \mathcal{O}_{X}
$$

where $\delta: \mathcal{A} \otimes_{\mathcal{O}_{X}} \bigwedge^{k} \mathcal{T} \rightarrow \mathcal{A} \otimes_{\mathcal{O}_{X}} \bigwedge^{k-1} \mathcal{T}$ is given by

$$
\begin{aligned}
\delta\left(a \otimes\left(t_{1} \wedge \ldots \wedge t_{k}\right)\right)=-\sum_{i=1}^{k}(-1)^{i} a t_{i} \otimes\left(t_{1} \wedge \ldots \wedge \hat{t_{i}} \wedge \ldots \wedge t_{k}\right) \\
\quad+\sum_{1 \leq i<j \leq k}(-1)^{i+j} a \otimes\left(\left[t_{i}, t_{j}\right] \wedge \ldots \wedge \hat{t_{i}} \wedge \ldots \wedge \hat{t_{j}} \wedge \ldots \wedge t_{k}\right),
\end{aligned}
$$

with $\hat{t_{i}}$ denoting omission. Notice that by (S3) the commutator

$$
\left[t_{i}, t_{j}\right]=\sigma\left(t_{i}\right) \sigma\left(t_{j}\right)-\sigma\left(t_{j}\right) \sigma\left(t_{i}\right)
$$

belongs to $\mathcal{A}^{(1)}$ and gives thus an element of $\mathcal{T}$. The augmentation $\epsilon$ is simply the $\mathcal{A}$-linear morphism specified by $1 \mapsto 1$.

LEMma 2.1.8. The augmented complex (2.1.7.1) gives a locally free left resolution of the $\mathcal{A}$-module $\mathcal{O}_{X}$.

Proof. Since $\mathcal{T}$ is locally free over $\mathcal{O}_{X}$, so are the terms of the resolution over $\mathcal{A}$. For exactness, one may check that the proof of the stronger statement (2.1.18) in Mebkhout [19] for ordinary $\mathcal{D}$-modules does not use assumptions that go beyond (S1) to (S4).

Remark 2.1.9. When $\mathcal{A}=\mathcal{D}_{X / S}$, the resolution is the usual Spencer resolution. In the case where $\mathcal{A}=\operatorname{Sym}^{\bullet} \mathcal{T}_{X / S}$, one can check that the resolution reduces to a Koszul complex, the exactness of which is easy to verify. by

Definition 2.1.10. The functor $\mathbf{D R}=\mathbf{D R}_{X / S}: \mathbf{D}_{q c}^{b}(\mathcal{A}) \rightarrow \mathbf{D}^{b}\left(f^{-1} \mathcal{O}_{S}\right)$ given

$$
\mathbf{D R}(\mathcal{M})=\mathbf{R} \mathcal{H o m}_{\mathcal{A}}\left(\mathcal{O}_{X}, \mathcal{M}\right)
$$

is called the (generalised) de Rham functor.

Proposition 2.1.11. Let $\mathcal{M}$ be an $\mathcal{A}$-module.

1. If $\mathcal{A}=\mathcal{D}_{X / S}$, the complex $\mathbf{D R}(\mathcal{M})$ is the usual relative de Rham complex

$$
0 \rightarrow \mathcal{M} \stackrel{\nabla}{\rightarrow} \mathcal{M} \otimes \Omega_{X / S}^{1} \stackrel{\nabla}{\rightarrow} \mathcal{M} \otimes \Omega_{X / S}^{2} \stackrel{\nabla}{\rightarrow} \cdots
$$

with $\nabla(m \otimes \alpha)=\nabla m \wedge \alpha-(-1)^{\operatorname{deg} \alpha} m \otimes d \alpha$

2. If $\mathcal{A}=\operatorname{Sym}^{\bullet} \mathcal{T}_{X / S}$, the complex $\mathbf{D R}((\mathcal{E}, \theta))$ is

$$
0 \rightarrow \mathcal{E} \stackrel{\theta}{\rightarrow} \mathcal{E} \otimes \Omega_{X / S}^{1} \stackrel{\theta}{\rightarrow} \mathcal{E} \otimes \Omega_{X / S}^{2} \stackrel{\theta}{\rightarrow} \cdots,
$$

where $\theta(e \otimes \alpha)=\theta(e) \wedge \alpha$. In particular, this complex is $\mathcal{O}_{X}$-linear. 
Proof. We use the generalised Spencer resolution (2.1.7) of $\mathcal{O}_{X}$ to compute the de Rham object $\mathbf{D R}(\mathcal{M})=\mathbf{R} \mathcal{H o m}_{\mathcal{A}}\left(\mathcal{O}_{X}, \mathcal{M}\right)$. Part (1) is well-known, see any of the references on $\mathcal{D}_{X}$-modules. For (2), we notice that in (2.1.7.2) the terms involving commutators $\left[t_{i}, t_{j}\right]$ vanish for $\mathcal{A}=\operatorname{Sym}^{\bullet} \mathcal{T}_{X / S}$. Thus the second term $-(-1)^{\operatorname{deg} \alpha} m \otimes$ $d \alpha$ of the formula in case (1) vanishes. $\square$

REmark 2.1.12. Let $\mathcal{M}$ be a $\mathcal{D}_{X}$-module. It follows from (2.1.11) that the hypercohomology $\mathbf{H}^{\bullet}(X, \mathbf{D R}(\mathcal{M}))$ is precisely the cohomology of $X$ with coefficients the local system $\mathcal{L}(\mathcal{M})$ of horizontal sections of the flat connection, denoted by $H_{d R}^{\bullet}(X, \mathcal{M})$ in Simpson [23]. Similarly, for a Higgs bundle $\mathrm{E}=(\mathcal{E}, \theta)$, the hypercohomology $\mathbf{H}^{\bullet}(X, \mathbf{D R}(\mathrm{E}))$ is Simpson's Dolbeault cohomology $H_{D o l}^{\bullet}(X, \mathrm{E})$.

Proposition 2.1.13. If $f: X \rightarrow S$ is proper, then $\mathbf{R} f_{*} \mathbf{D R}(\mathcal{M})$ ) is coherent for an $\mathcal{O}_{X}$-coherent $\mathcal{A}$-module $\mathcal{M}$.

Proof. This follows from the first hypercohomology spectral sequence with firstdegree terms

$$
E_{1}^{p q}=R^{q} f_{*} \mathbf{D R}(\mathcal{M})^{p} \Rightarrow \mathbf{R}^{p+q} f_{*} \mathbf{D R}(\mathcal{M}) .
$$

Indeed, each term of $\mathbf{D R}(\mathcal{M})$ is a coherent $\mathcal{O}_{X}$-module, and hence each sheaf $R^{q} f_{*} \mathbf{D R}(\mathcal{M})^{p}$ is $\mathcal{O}_{S}$-coherent. On the other hand, since $\mathbf{D R}(\mathcal{M})$ is $f^{-1} \mathcal{O}_{S}$-linear, the differentials in (2.1.13.1) are $\mathcal{O}_{S}$-linear, whence the proposition.

2.2. $\lambda$-connections. We resume and complement the treatment of $\lambda$-connections in Simpson [26]. $\lambda$-connections interpolate between bundles with flat connection and Higgs bundles; their description as suitable Simpson modules relates this to deformation of $\mathcal{D}_{X}$ to its associated graded algebra. The theory was first outlined in Deligne [8] and it was developed by Simpson in [26]. What follows is essentially a quick review of Simpson's results needed in the sequel.

In this subsection $X$ is a smooth projective $\mathbf{C}$-scheme and thus is equipped with a very ample line bundle $\mathcal{O}_{X}(1)$. The first Chern class $c_{1}\left(\mathcal{O}_{X}(1)\right)$ is the polarisation $[\omega]$ of $X$. If $X$ is given the Kähler metric induced by the projective embedding, then the polarisation class is represented by the Kähler form $\omega_{X}$.

Definition 2.2.1. Let $\lambda: S \rightarrow \mathbf{A}^{1}$ be a morphism of schemes, and let $\mathcal{E}$ be a locally free $\mathcal{O}_{X \times S}$-module. A $\lambda$-connection on $\mathcal{E}$ is a morphism of sheaves

$$
\nabla: \mathcal{E} \rightarrow \mathcal{E} \otimes_{\mathcal{O}} \Omega_{X \times S / S}^{1},
$$

satisfying the following conditions:

1. $\nabla(a e)=\lambda e \otimes d a+a \nabla(e) \quad$ "Leibnitz rule"

2. $\nabla^{2}=\nabla \circ \nabla=0$,

where $\nabla$ is extended to a map $\mathcal{E} \otimes \Omega_{X \times S / S}^{1} \rightarrow \mathcal{E} \otimes \Omega_{X \times S / S}^{2}$ by the rule $\nabla(e \otimes \alpha)=$ $\nabla(e) \wedge \alpha+\lambda \cdot e \otimes d \alpha$.

If $\lambda$ is the constant map with value $c \in \mathbf{C}$, then we call a $\lambda$-connection also a (family of) $c$-connection(s). It is clear that a 1-connection is just a (relative) flat connection. Similarly, for $\lambda=0$, the first condition says that $\nabla$ is $\mathcal{O}_{X \times S}$-linear and the second condition is simply $[\nabla, \nabla]=0$; in other words, a bundle with a 0 -connection is precisely a Higgs bundle. Notice that the condition $[\nabla, \nabla]=0$ is vacuous if $X$ is a curve. 
2.2.2. Define the sheaf of algebras $\Lambda$ on $X \times \mathbf{A}^{1}$ to be the subsheaf of $\operatorname{pr}_{X}^{*} \mathcal{D}_{X}$ generated by sections of the form

$$
\sum_{k} t^{k} u_{k}
$$

where the $u_{k}$ are sections of $\mathcal{D}_{X}^{(k)}$ and $t$ is the linear coordinate on $\mathbf{A}^{1}$. Then $\left.\Lambda\right|_{X \times\{t\}}$ is isomorphic to $\mathcal{D}_{X}$ for any $t \neq 0$, and $\left.\Lambda\right|_{X \times\{0\}}$ is isomorphic to $\operatorname{gr}^{\bullet} \mathcal{D}_{X}$ (see the discussion in Section 5 of Simpson [26]). So $\Lambda$ gives a deformation of $\mathcal{D}_{X}$ to $\operatorname{Sym}^{\bullet} \mathcal{T}_{X}$. Furthermore, $\Lambda$ is a Simpson ring on $X \times \mathbf{A}^{1}$ over $\mathbf{A}^{1}$, with $\mathbf{g r}^{1} \Lambda=\operatorname{pr}_{X}^{*} \mathcal{T}_{X}$ (Simpson [24] p. 81).

2.2.3. Let us consider the situation of (2.2.1). For $\lambda: S \rightarrow \mathbf{A}^{1}$, the pull-back $\Lambda_{\lambda}=\left(1_{X} \times \lambda\right)^{*} \Lambda$ on $X \times S$ is also a Simpson ring over $S$, with $\operatorname{gr}^{1} \Lambda_{\lambda}=\operatorname{pr}_{X}^{*} \mathcal{T}_{X}$. It is easy to see that to give a locally free $\mathcal{O}_{X \times S}$-module a $\lambda$-connection is precisely the same thing as to give it a structure of $\Lambda_{\lambda}$-module.

2.2.4. Recall that the slope $\mu(\mathcal{E})$ of a locally free $\mathcal{O}_{X}$-module $\mathcal{E}$ on manifold $X$ with polarisation given by a Kähler form $\omega_{X}$ is $\mu(\mathcal{E})=\operatorname{deg} \mathcal{E} / \operatorname{rank} \mathcal{E}$, where $\operatorname{deg} \mathcal{E}=$ $\int_{X} c_{1}(\mathcal{E}) \wedge \omega_{X}^{\operatorname{dim} X-1}$. Thus if $\operatorname{dim} X \geq 2$, the degree and hence stability of $\mathcal{E}$ depends on the polarisation $\left[\omega_{X}\right]$.

Definition 2.2.5. Let $a \in \mathbf{A}^{1}$. Then a sheaf $\mathcal{E}$ on $X$ with an $a$-connection $\nabla$ is stable (resp. semi-stable) if for each locally free subsheaf $\mathcal{F} \subset \mathcal{E}$ stable under $\nabla$ we have $\mu(\mathcal{F})<\mu(\mathcal{E})($ resp. $\mu(\mathcal{F}) \leq \mu(\mathcal{E}))$.

Let $\lambda: S \rightarrow \mathbf{A}^{1}$ be a morphism. Then a sheaf $\mathcal{E}$ on $X \times S$ with a $\lambda$-connection $\nabla$ is stable (resp. semi-stable) if $\mathcal{E}$ is flat over $S$ and $\left(\mathcal{E}_{s}, \nabla_{s}\right)$ is a stable (resp. semi-stable) sheaf with $\lambda(s)$-connection for all $s \in S$.

REMARK 2.2.6. For $a \neq 0$ each bundle $\mathcal{E}$ with an $a$-connection is semi-stable; indeed, the slope of a subsheaf preserved by a flat connection is necessarily the same as the slope of $\mathcal{E}$, i.e., zero.

Theorem 2.2.7 (Simpson). Consider the functor $M: \mathfrak{S c h}_{/ \mathbf{A}^{1}} \rightarrow$ Set which to each $\mathbf{A}^{1}$-scheme $\lambda: S \rightarrow \mathbf{A}^{1}$ associates the set of isomorphism classes of pairs $(\mathcal{E}, \nabla)$, where $\mathcal{E}$ is a locally free sheaf of rank $n$ on $X \times S$ having vanishing (rational) Chern classes along the fibres of $\lambda$, and $\nabla$ is a $\lambda$-connection making $(\mathcal{E}, \nabla)$ semi-stable. Then:

1. There is a quasi-projective moduli space $\mathbf{M}_{H o d}(X, n)$ for $M$, i.e., a quasiprojective scheme that universally co-represents $M$.

2. $\mathbf{M}_{H o d}(X, n)$ has a natural projection $\pi$ to $\mathbf{A}^{1}$, and the geometric points of the fibre $\pi^{-1}\{a\}$ correspond bijectively to Jordan equivalence classes of semistable bundles with a-connections on $X$.

Proof. Apply Theorem 4.7. of Simpson [24] to the sheaf of rings $\Lambda$ of (2.2.2) on $X \times \mathbf{A}^{1}$. This gives disjoint moduli spaces for $\mathbf{A}^{1}$-flat $\Lambda$-modules with fixed normalised Hilbert polynomials. Let $P_{0}$ be the Hilbert polynomial of $\mathcal{O}_{X}$, and let $\mathbf{M}^{n P_{0}}$ be the moduli space corresponding to $n P_{0}$. Consider the subfunctor $M_{0}^{n P_{0}}$ of $M^{n P_{0}}$ which classifies the relative $\lambda$-connections on $X \times S$ with vanishing Chern classes along the fibres $X \times\{s\}$. Since the Chern classes $c_{i}$ of a flat family $\left(\mathcal{E}_{s}\right)_{s \in S}$ of coherent sheaves on a scheme $X$ smooth and projective over $S$ (considered as sections of the relative de Rham cohomology $\mathbf{R}^{2 i} \operatorname{pr}_{S *} \Omega_{X / S}^{\bullet}$ ) are horizontal with respect to the Gauss-Manin 
connection, the vanishing of $c_{i}\left(\mathcal{E}_{s}\right)$ depends only on the connected component of $S$ containing $s$. It follows that the functor $M_{0}^{P_{0}}$ is universally co-represented by a union of connected components of $\mathbf{M}^{n P_{0}}$; this open subset is $\mathbf{M}_{H o d}(X, n)$.

REMARKS 2.2.8.

1. The fibres of $\mathbf{M}_{H o d}(X, n)$ over 0 and 1 are respectively the moduli spaces $\mathbf{M}_{D o l}(X, n)$ of semi-stable Higgs bundles and $\mathbf{M}_{d R}(X, n)$ of flat bundles.

2. The moduli space $\mathbf{M}_{D o l}(X, 1)$ of rank-1 Higgs bundles on a smooth projective curve $X$ can be identified with $\mathrm{J}(X) \times H^{0}\left(X, \Omega_{X}^{1}\right)$, or the (trivial) cotangent bundle of the Jacobian.

3. Let $X$ be a curve; choosing a principal polarisation of the Jacobian $\mathrm{J}(X)$ lets us identify the Jacobian with its dual. Given this identification, the moduli space $\mathbf{M}_{d R}(X, 1)$ is identified with Grothendieck's universal vector extension $\mathrm{J}(X)^{\natural}$ of $\mathrm{J}(X)$, see Mazur-Messing [18] or Laumon [17]. In particular, for a scheme $S$, the (algebraic group) extensions of $\mathrm{J}(X) \times S$ by a vector bundle $\mathbf{V}\left(\mathcal{E}^{\vee}\right)$ on $S$ correspond bijectively to the morphisms $H^{0}\left(X, \omega_{X}\right) \otimes \mathcal{O}_{S} \rightarrow \mathcal{E}$. We obtain the following description of the moduli space $\mathbf{M}_{H o d}(X, 1)$ : there is an exact sequence

$$
0 \rightarrow \mathbf{V}\left(H^{0}\left(X, \omega_{X}\right)^{\vee}\right) \times \mathbf{A}^{1} \rightarrow \mathbf{M}_{H o d}(X, 1) \stackrel{\pi}{\rightarrow} \mathrm{J}(X) \times \mathbf{A}^{1} \rightarrow 0
$$

of group schemes over $\mathbf{A}^{1}$, where $\pi$ takes a $\lambda$-connection to its underlying line bundle. This extension is now just the "push-out" of the universal extension by the multiplication-by- $\lambda \in \mathbf{A}^{1}$ morphism

$$
[\lambda]: H^{0}\left(X, \omega_{X}\right) \otimes \mathcal{O}_{\mathbf{A}^{1}} \rightarrow H^{0}\left(X, \omega_{X}\right) \otimes \mathcal{O}_{\mathbf{A}^{1}}
$$

3. Hyper-Kähler structure. We first review the existence of a hyper-Kähler structure on the base manifold $\mathbf{M}_{D o l}(X, 1)$ of the Fourier transform of a Higgs bundle on $X$. This is a consequence of the Hitchin-Simpson correspondence, relating a Higgs field to a flat connection in the same underlying vector bundle and leading to the existence of two different moduli space structures on the same underlying set.

A hyper-Kähler manifold has an associated twistor space, which we shall use to characterise autodual connections later on. In subsection 3.2 we review Deligne's approach to describing the twistor space of $\mathbf{M}_{D o l}(X, n)$ in terms of moduli spaces of $\lambda$-connections.

3.1. Harmonic metrics and the hyper-Kähler structure of $\mathbf{M}_{d R}(X, n)$. We continue to assume that $X$ is a smooth projective variety, and hence $a$ fortiori a compact Kähler manifold. Let $\omega$ be the the corresponding Kähler form. What follows is essentially a resumé of the results of Hitchin, Donaldson, Simpson, Corlette and Fujiki, made explicit to the extent that the details of the arguments are needed in the sequel.

3.1.1. Let $\mathcal{E}$ be a locally free $\mathcal{O}_{X}$-module with a flat connection $\nabla$, and let $E$ be the underlying smooth complex vector bundle of $\mathcal{E}$. We continue to denote the corresponding flat connection on $E$ by $\nabla$; it has the decomposition $\nabla=\nabla^{\prime}+\nabla^{\prime \prime}$ into operators of type $(1,0)$ and $(0,1)$ respectively. Assume that $E$ is equipped with a Hermitean metric $h$. Then we define operators $\delta^{\prime}$ and $\delta^{\prime \prime}$ to be the unique operators of types $(1,0)$ and $(0,1)$ such that $\nabla^{\prime}+\delta^{\prime \prime}$ and $\delta^{\prime}+\nabla^{\prime \prime}$ are connections preserving the 
metric $h$. We set

$$
\begin{array}{ll}
\partial=\partial_{h}=\left(\nabla^{\prime}+\delta^{\prime}\right) / 2 & \theta=\theta_{h}=\left(\nabla^{\prime}-\delta^{\prime}\right) / 2 \\
\bar{\partial}=\bar{\partial}_{h}=\left(\nabla^{\prime \prime}+\delta^{\prime \prime}\right) / 2 & \bar{\theta}=\bar{\theta}_{h}=\left(\nabla^{\prime \prime}-\delta^{\prime \prime}\right) / 2 .
\end{array}
$$

Notice that

$$
\nabla^{\prime}=\partial+\theta \quad \text { and } \quad \nabla^{\prime \prime}=\bar{\partial}+\bar{\theta} .
$$

Since $\nabla$ is flat, $\nabla^{\prime 2}=\nabla^{\prime \prime 2}=\nabla^{\prime} \nabla^{\prime \prime}+\nabla^{\prime \prime} \nabla^{\prime}=0$. This implies for the operators induced by $h$ that $\delta^{\prime 2}=\delta^{\prime \prime 2}=\delta^{\prime} \delta^{\prime \prime}+\delta^{\prime \prime}+\delta^{\prime}=0$. Hence $\bar{\partial}_{h}$ is a complex structure operator in $E$ if and only if

$$
\bar{\partial}_{h}^{2}=\nabla^{\prime \prime} \delta^{\prime \prime}+\delta^{\prime \prime} \nabla^{\prime \prime}=0
$$

If this is the case, then $\theta_{h}$ is holomorphic with respect to $\bar{\partial}_{h}$ precisely when

$$
\bar{\partial}_{h}\left(\theta_{h}\right)=\nabla^{\prime} \delta^{\prime \prime}+\delta^{\prime \prime} \nabla^{\prime}-\nabla^{\prime \prime} \delta^{\prime}-\delta^{\prime} \nabla^{\prime \prime}=0 .
$$

Finally, for $\theta_{h}$ to be a Higgs field it needs to satisfy

$$
\theta_{h}^{2}=-\nabla^{\prime} \delta^{\prime}-\delta^{\prime} \nabla^{\prime}=0 .
$$

The sum $G_{h}=\bar{\partial}_{h}^{2}+\bar{\partial}_{h}\left(\theta_{h}\right)+\theta_{h}^{2}$ of the operators above is an $\operatorname{End}(E)$-valued differential 2 -form, the pseudo-curvature of the metric $h$ (with respect to $\nabla)$. Hence $\left(E, \bar{\partial}_{h}, \theta_{h}\right)$ is a Higgs bundle precisely when $G_{h}=0$. In this case we call the metric $h$ harmonic. Notice that the Higgs bundle structure on $E$ is completely specified by the operator $D_{E}=\bar{\partial}_{h}+\theta$.

Theorem 3.1.2 (Simpson). Let $(\mathcal{E}, \nabla)$ be a flat vector bundle on $X$ and let $E$ be the underlying smooth complex bundle.

1. There is in $E$ an (essentially unique) Hermitean metric $h$ with vanishing pseudo-curvature $G_{h}$ if and only if the monodromy representation

$$
\rho_{\nabla}: \pi_{1}(X, x) \rightarrow \mathbf{G L}\left(E_{x}\right)
$$

is semi-simple.

2. The construction of (3.1.1) establishes an equivalence between the category of flat bundles on $X$ with semisimple monodromy and the category of direct sums of stable Higgs bundles on $X$ with vanishing Chern classes.

Proof. For claim (1), let $\Lambda$ denote the adjoint of wedging with $\omega$. The equivalence of the existence of a metric $h$ such that $\Lambda G_{h}=0$ and the semi-simplicity of the monodromy representation is a deep analytic theorem of Corlette [7]. That $\Lambda G_{h}=0$ implies $G_{h}=0$ is Lemma 1.1. of Simpson [23].

Claim (2) is Corollary 1.3 of Simpson [23]. There is an inverse construction of a connection on $E$ starting from a Higgs bundle structure and a a metric $h$ on $E$, similar to the one in (3.1.1) (see ibid. p. 13). The existence of a metric $h$ making this connection flat if the Higgs bundle is a direct sum of stable Higgs bundles with vanishing Chern classes is a hard theorem of non-linear analysis in Simpson [22]. That this construction and the one of (3.1.1) are inverses to each other is esteblished on p. 13 of Simpson [23]. This establishes the action of the equivalence functor on objects. The functor acts trivially on morphisms of the underlying smooth bundle. More 
precisely, let $\left(E, \nabla_{E}\right)$ and $\left(F, \nabla_{F}\right)$ be two vector bundles with flat connections. Then the morphisms between them correspond precisely to the smooth sections of $E^{*} \otimes F$ killed by the natural flat connection $\nabla_{E^{*} \otimes F}$ on the tensor product. On the other hand, let $\left(E, \theta_{E}\right)$ and $\left(F, \theta_{F}\right)$ be the corresponding Higgs bundles; then $E^{*} \otimes F$ carries also a natural Higgs bundle structure (the Higgs field is $1 \otimes \theta_{F}-\theta_{E}^{t} \otimes 1$ ). Now it is easy to see that a section of $E^{*} \otimes F$ is a morphism of Higgs bundles precisely if it is killed by the operator $D_{E^{*} \otimes F}$ specified in (3.1.1). Moreover, the harmonic metrics on $E$ and $F$ relating their flat connections and Higgs bundles structures define a harmonic metric on $E^{*} \otimes F$ (ibid. p.14) which similarly relates the connection $\nabla_{E^{*} \otimes F}$ to the operator $D_{E^{*} \otimes F}$ specifying the Higgs bundle structure. But then it follows from ibid. Lemma 1.2. that if $\varphi$ is a section of $E^{*} \otimes F$, then $\nabla_{E^{*} \otimes F}(\varphi)=0$ if and only if $D_{E^{*} \otimes F}(\varphi)=0$. Hence precisely the same morphisms of the underlying smooth bundles underlie the morphisms between the flat bundles and the corresponding Higgs bundles. $\square$

Remark 3.1.3. We keep the assumptions of (3.1.1). Let $\bar{X}$ be the complex conjugate manifold of $X$. Conjugation switches the roles of $(1,0)$ forms and $(0,1)$ forms and the roles of $\partial$ and $\bar{\partial}$. It follows that in the construction (3.1.1), $\partial_{h}$ and $\bar{\partial}_{h}$ get exchanged, as do $\theta_{h}$ and $\bar{\theta}_{h}$.

We may then ask if $\partial_{h}$ is a holomorphic structure operator for $E$ and whether $\bar{\theta}_{h}$ is a Higgs field. It is immediate that the corresponding pseudo-curvature operator is $-G_{h}$. It follows that $\left(E, \partial_{h}, \bar{\theta}_{h}\right)$ is a Higgs bundle precisely when $h$ is harmonic or in other words when $\left(E, \bar{\partial}_{h}, \theta_{h}\right)$ is a Higgs bundle. Clearly the Higgs bundle $\left(E, \partial_{h}, \bar{\theta}_{h}\right)$ on $\bar{X}$ is stable precisely when $\left(E, \bar{\partial}_{h}, \theta_{h}\right)$ is stable on $X$.

THEOREM 3.1.4. The equivalence of categories of (3.1.2) induces a homeomorphism between the moduli spaces $\mathbf{M}_{d R}(X, n)$ and $\mathbf{M}_{D o l}(X, n)$. Restricted to the smooth loci of the moduli spaces it induces a real-analytic isomorphism between the manifolds $\mathbf{M}_{d R}(X, n)^{\mathrm{sm}}$ and $\mathbf{M}_{D o l}(X, n)^{\mathrm{sm}}$.

Proof. For the homeomorphism see Theorem 7.18. of Simpson [25]. By Theorem (8.3.1) in Fujiki [11] the complex manifolds $\mathbf{M}_{d R}(X, n)^{\mathrm{sm}}$ and $\mathbf{M}_{D o l}(X, n)^{\mathrm{sm}}$ both belong to same hyper-Kähler family of complex structures on the underlying space. Hence the real-analyticity follows from Proposition 6.5. of Verbitsky [29].

EXAMPLE 3.1.5. Let $X$ be a smooth complete curve of genus $g$, the case that is the focus of our interest in applications to the Fourier transform. We notice first that the abelianisation of $\pi_{1}(X)$ is $\mathbf{Z}^{2 g}$, and hence the monodromy of a flat connection in a line bundle is specified by $2 g$ non-zero complex numbers. Thus by the RiemannHilbert correspondence $\mathbf{M}_{d R}(X, 1)$ is $\mathbf{G}_{m}^{2 g}=\left(\mathbf{C}^{*}\right)^{2 g}$. On the other hand, it is clear that $\mathbf{M}_{D o l}(X, 1)=\mathrm{J}(X) \times H^{0}\left(X, \Omega_{X}^{1}\right)$.

The underlying smooth complex vector bundle for all flat line bundles classified by $\mathbf{M}_{d R}(X, 1)$ is the trivial line bundle $L=X \times \mathbf{C}$. The canonical Hermitean product metric on $L$ is seen to be harmonic for all flat line bundles. But then one sees from the construction of (3.1.1) that Higgs bundles with zero Higgs field $\theta$ correspond precisely to unitary connections. Thus the homeomorphism $\mathbf{M}_{D o l}(X, 1) \rightarrow \mathbf{M}_{d R}(X, 1)$ of (3.1.4) takes $\mathrm{J}(X)$ bijectively to $\mathbf{U}(1)^{2 g} \subset\left(\mathbf{C}^{*}\right)^{2 g}$.

Let $\nabla$ be the flat connection corresponding to a holomorphic line bundle $\mathcal{L}=$ $(L, \bar{\partial})$. Then the connection corresponding to $(\mathcal{L}, \theta)$ is $\nabla+\theta+\bar{\theta}$. The monodromy of $\nabla+\theta+\bar{\theta}$ around a generator $\gamma_{i}$ of $\pi_{1}(X)$ is given by

$$
\operatorname{Mon}_{\gamma_{i}}(\nabla+\theta+\bar{\theta})=\operatorname{Mon}_{\gamma_{i}}(\nabla) \exp \left(-\int_{\gamma_{i}} \theta+\bar{\theta}\right) \text {. }
$$


Hence the homeomorphism $\mathbf{M}_{D o l}(X, 1) \rightarrow \mathbf{M}_{d R}(X, 1)$ of (3.1.4) is a homomorphism $\mathrm{J}(X) \times H^{0}\left(X, \Omega_{X}^{1}\right) \rightarrow \mathbf{U}(1)^{2 g} \times\left(\mathbf{R}_{+}^{*}\right)^{2 g}$, and gives us a natural non-holomorphic "polar" coordinate system on $\mathbf{M}_{d R}(X, 1)$.

3.1.6. Let $\mathrm{M}$ be the differentiable manifold underlying the smooth loci of both $\mathbf{M}_{d R}(X, n)$ and $\mathbf{M}_{D o l}(X, n)$. Then $M$ has two complex structures $I$ and $J$ given by $\mathbf{M}_{d R}(X, n)$ and $\mathbf{M}_{D o l}(X, n)$ respectively. The tangent space at $\mathcal{E}$ of $\mathbf{M}_{d R}(X, n)$ (resp. $\mathrm{E}$ of $\left.\mathbf{M}_{D o l}(X, n)\right)$ is isomorphic to $H^{1}(X, \mathbf{D R}(\mathcal{E}))=H_{d R}^{1}(X, \mathcal{E})\left(\operatorname{resp} \cdot \mathbf{H}^{1}(\mathbf{D R}(\mathrm{E}))=\right.$ $\left.H_{D o l}^{1}(X, \mathrm{E})\right)$ - see sections 8 and 9 in Fujiki [11] or p. 88 in Hitchin [12]. Both cohomology spaces can be described as spaces of suitable harmonic forms and hence come with $L^{2}$-metrics. These give $M$ two Riemannian metrics $g_{d R}$ and $g_{D o l}$, which in fact agree up to multiplication by a constant.

TheOREM 3.1.7. $M$ equipped with the metric $g_{d R}$ (or $g_{D o l}$ ) and the complex structures $I, J$ and $K=I J$ is a hyper-Kähler manifold.

In other words, the complex structures $I, J$ and $K$ satisfy the quaternionic identities

$$
I^{2}=J^{2}=K^{2}=I J K=-1,
$$

and $g_{d R}$ (resp. $g_{D o l}$ ) is Kähler with respect to $I, J$ and $K$.

Proof. For the case where $X$ is a curve, see Hitchin [12]; the general case is Theorem (8.3.1) in Fujiki [11]. $\square$

3.2. Twistor space of $\mathbf{M}_{D o l}(X, n)$. We recall the definition of the twistor space of a hyper-Kähler manifold and explain P. Deligne's description of the twistor spaces of the moduli spaces $\mathbf{M}_{d R}(X, n)$ and $\mathbf{M}_{D o l}(X, n)$.

3.2.1. Let $(M, h)$ be a hyper-Kähler manifold with complex structures $I, J$ and $K$ satisfying the relation $I J K=I^{2}=J^{2}=K^{2}=-1$ and with respect to which the metric $h$ is Kähler. We identify the unit sphere $S^{2} \subset \mathbf{R}^{3}$ with $\mathbf{P}_{\mathbf{C}}^{1}$. For any $z=(a, b, c) \in S^{2}$ we get an almost complex structure $I_{z}=a I+b J+c K$ on $M$. It is straightforward to check that $I_{z}$ is integrable and that $h$ is Kähler with respect to $I_{z}$.

The tangent space $T_{(m, z)}\left(M \times \mathbf{P}_{\mathbf{C}}^{1}\right)$ splits as $T_{m} M \oplus T_{z} \mathbf{P}_{\mathbf{C}}^{1}$. Give it the almost complex structure

$$
\left(\begin{array}{cc}
I_{z} & 0 \\
0 & I_{\mathbf{P}^{1}}
\end{array}\right)
$$

where $I_{\mathbf{P}^{1}}$ is the standard complex structure of $\mathbf{P}^{1}$. We have the following theorem of Atiyah, Hitchin and Singer (see Salamon [21] and Hitchin et al. [14]):

TheOREM 3.2.2. The almost complex structure above is integrable. The projection $\operatorname{pr}_{\mathbf{P}}: \mathbf{T w}(M) \rightarrow \mathbf{P}_{\mathbf{C}}^{1}$ is holomorphic, whereas the projection $\operatorname{pr}_{M}: \mathbf{T w}(M) \rightarrow M$ is only real analytic.

Definition 3.2.3. The complex manifold $\mathbf{T w}(M)=M \times \mathbf{P}_{\mathbf{C}}^{1}$ is called the twistor space of $M$. The holomorphic sections of the form $\tilde{m}: \mathbf{P}^{1} \rightarrow\{m\} \times \mathbf{P}^{1}$ for $m \in M$ are called horizontal twistor lines.

For the moduli spaces $\mathbf{M}_{d R}(X, n)$ and $\mathbf{M}_{D o l}(X, n)$ there is a complex-analytic description of the twistor space, due to P. Deligne [8], worked out in Simpson [26]: 
3.2.4. The multiplicative group scheme $\mathbf{G}_{m}$ acts on $\mathbf{A}^{1}$ by multiplication. This action lifts to an action of $\mathbf{G}_{m}$ on $\mathbf{M}_{H o d}(X, n)$ over $\mathbf{A}^{1}$ : if $t \in \mathbf{G}_{m}(S)$ and $(\mathcal{E}, \nabla)$ is a $\lambda$-connection on $X \times S$, then $(\mathcal{E}, t \nabla)$ is a $t \lambda$-connection, and this action gives an isomorphism $\mathbf{M}_{H o d}(X, n) \rightarrow \mathbf{M}_{H o d}(X, n)$ covering $[t]: \mathbf{A}^{1} \rightarrow \mathbf{A}^{1}$. In particular, this action identifies the fibres of $\mathbf{M}_{\text {Hod }}(X, n)$ over any $\lambda, \lambda^{\prime} \neq 0$ - they are all isomorphic to $\mathbf{M}_{d R}(X, n)$. Thus we have the isomorphism

$$
\mathbf{M}_{H o d}(X, n) \times \mathbf{A}^{1} \mathbf{G}_{m} \cong \mathbf{M}_{d R}(X, n) \times \mathbf{G}_{m} .
$$

On the other hand, by the "Riemann-Hilbert correspondence" associating to a flat connection its monodromy representation, $\mathbf{M}_{d R}(X, n)$ is canonically complexanalytically (but not algebraically, see Simpson [25]) isomorphic to the moduli space $\mathbf{M}_{B}(X, n)$ of representations

$$
\rho: \pi_{1}(X) \rightarrow \mathbf{G L}(n, \mathbf{C}) .
$$

Let $\check{\rho}$ denote the contragredient conjugate representation

$$
\check{\bar{\rho}}(\gamma)={ }^{t} \overline{\rho(\gamma)}^{-1},
$$

i.e., the complex conjugate of the transposed inverse. Then $\rho \mapsto \check{\bar{\rho}}$ induces a complex anti-holomorphic involution $\tau$ of $\mathbf{M}_{B}(X, n)$, hence an anti-holomorphic involution $\tau^{\prime}$ of $\mathbf{M}_{d R}(X, n)$. Let $\sigma: \mathbf{P}^{1} \rightarrow \mathbf{P}^{1}$ denote the antipodal map, which is also an anti-holomorphic involution (indeed the real structure of $\mathbf{P}^{1}$ without real points). Restricted to $\mathbf{G}_{m}(\mathbf{C})=\mathbf{C}^{*}$, it is given by $z \mapsto-\bar{z}^{-1}$.

Putting these together, we get an anti-holomorphic involution $\sigma^{\prime}$ of $\mathbf{M}_{d R}(X, n) \times$ $\mathbf{G}_{m}$ by

$$
\sigma^{\prime}(u, t)=\left(\tau^{\prime}(u), \sigma(t)\right)
$$

But with the identifications above, this gives an isomorphism

$$
\sigma^{\prime}: \mathbf{M}_{H o d}(X, n) \times_{\mathbf{A}^{1}} \mathbf{G}_{m} \rightarrow \overline{\mathbf{M}_{H o d}(X, n) \times{ }_{\mathbf{A}^{1}} \mathbf{G}_{m}}
$$

between the complex conjugate schemes. Let $T$ be the scheme obtained by glueing $\mathbf{M}_{\text {Hod }}(X, n)$ to $\overline{\mathbf{M}_{\text {Hod }}(X, n)}$ over $\mathbf{G}_{m}$ using $\sigma^{\prime}$. Since $\mathbf{P}^{1}$ is glued from $\mathbf{A}^{1}$ and $\overline{\mathbf{A}^{1}}$ using $\sigma$, the projection $\mathbf{M}_{H o d}(X, n) \rightarrow \mathbf{A}^{1}$ gives a projection $\pi: T \rightarrow \mathbf{P}^{1}$.

THEOREM 3.2.5. The smooth locus $T^{\mathrm{sm}}$ of $T^{\mathrm{an}}$ is complex-analytically isomorphic to the twistor space $\mathbf{T w}\left(\mathbf{M}_{D o l}(X, n)^{\mathrm{sm}}\right)$ of the smooth locus of $\mathbf{M}_{D o l}(X, n)$.

Proof. Theorem 4.2 of Simpson [26].

Proposition 3.2.6. Let $\mathrm{E}=(\mathcal{E}, \theta)$ be a stable Higgs bundle with vanishing Chern classes, and let $\rho: \pi_{1}(X) \rightarrow \mathbf{G L}(n, \mathbf{C})$ be the monodromy of the associated flat bundle. Then the Higgs bundle on $X$ corresponding to the flat connection with monodromy $\tau(\rho)=\check{\bar{\rho}}$ is $(\mathcal{E},-\theta)$.

Proof. This proof is due to Simpson. We choose a harmonic metric $h$ in the underlying smooth bundle $E$. Let $\bar{E}$ be the complex conjugate bundle. Let $\bar{\partial}$ be the holomorphic structure of $\mathcal{E}$. In (3.1.3) we made $E$ into a Higgs bundle on $\bar{X}$ with same associated flat bundle using $\partial_{h}$ as the holomorphic structure operator and $\bar{\theta}_{h}$ as the Higgs field. By conjugating again, we can make $\bar{E}$ into a Higgs bundle $\overline{\mathrm{E}}$ on $X$. 
Indeed, sections of $\bar{E}$ are of the form $\bar{e}$ for $e \in \Gamma(X, E)$, and we take $\bar{e} \mapsto \overline{\partial_{h}(e)}$ to be holomorphic structure operator, and $\bar{e} \mapsto \bar{\theta}_{h}(e)$ to be the Higgs field. Moreover, we equip $\bar{E}$ with the induced metric $(\bar{e} \mid \bar{f})=(e \mid f)_{h}$; this metric is clearly harmonic. The flat connection associated to $\overline{\mathrm{E}}$ is seen to be $\bar{\nabla}(\bar{e})=\overline{\nabla(e)}$, where $\nabla$ is the flat connection associated to $\mathrm{E}$. The monodromy representation of $(\bar{E}, \bar{\nabla})$ is the complex conjugate of the monodromy representation of $(E, \nabla)$.

The metric gives a bundle map $M: E \otimes \bar{E} \rightarrow X \times \mathbf{C}$ by $M(e \otimes \bar{f})=(e \mid f)_{h}$. Let $\mathbf{F}=(E, \bar{\partial},-\theta)$, and consider the Higgs bundle $\mathbf{F} \otimes \overline{\mathbf{E}}$. We have

$$
M(\bar{\partial}(e), \bar{f})+M\left(e, \overline{\partial_{h}(f)}\right)=(\bar{\partial}(e) \mid f)_{h}+\left(e \mid \partial_{h}(f)\right)_{h}=\bar{\partial}\left((e \mid f)_{h}\right)
$$

since $\bar{\partial}+\partial_{h}$ is compatible with $h$. But this means that $M$ is a morphism of holomorphic bundles. Similarly,

$$
M\left(\theta_{\mathrm{F} \otimes \overline{\mathrm{E}}}(e \otimes \bar{f})\right)=-(\theta(e) \mid f)_{h}+\left(e \mid \bar{\theta}_{h}(f)\right)_{h}=0
$$

since $\theta$ and $\bar{\theta}_{h}$ are adjoint with respect to $h$. But this means that $M$ is a morphism of Higgs bundles

$$
M: \mathrm{F} \otimes \overline{\mathrm{E}} \rightarrow\left(\mathcal{O}_{X}, 0\right)
$$

$M$ comes from the metric and hence it is a perfect pairing, which shows that $\mathrm{F}$ is the dual Higgs bundle of $\bar{E}$. Since the correspondence between Higgs bundles and flat bundles preserves duality, the monodromy representation associated to $F$ is the contragredient of the representation associated to $\bar{E}$. But this is precisely the representation $\gamma \mapsto{ }^{t} \overline{\rho(\gamma)}^{-1}$.

3.2.7. The horizontal twistor lines are described in this framework by harmonic metrics on the underlying bundles of the $\lambda$-connections: Let $m \in \mathbf{M}_{d R}(X, n)$ correspond to a semi-simple flat bundle $(E, \nabla)$, and consider the decomposition (3.1.1.1) of $\nabla$ issuing from the harmonic metric. For $\lambda \in \mathbf{A}^{1}(\mathbf{C})$ define in $E$ an almost complex structure

$$
\bar{\partial}_{\lambda}=\bar{\partial}+\lambda \bar{\theta}
$$

and an operator

$$
\nabla_{\lambda}=\lambda \partial+\theta
$$

Then $\bar{\partial}_{\lambda}$ is integrable and $\nabla_{\lambda}$ is a $\lambda$-connection in $\left(E, \bar{\partial}_{\lambda}\right)$. This family is clearly holomorphic in $\lambda$, and so we have a relative $\lambda$-connection on $\mathbf{A}^{1} \times X / \mathbf{A}^{1}$ and thus a section $\sigma$ of the canonical map $\lambda: \mathbf{M}_{d R}(X, n) \rightarrow \mathbf{A}^{1}$.

We produce similarly a conjugate line in $\overline{\mathbf{M}_{\text {Hod }}(X, 1)}$, and we have to check that these glue together under $\sigma^{\prime}$ to give the horizontal twistor line. The verification of this is straightforward, see pp.233-234 in Simpson [26].

Proposition 3.2.8. If $X$ is a smooth projective curve, then $\mathbf{M}_{H o d}(X, 1)$ is a fine moduli space, i.e., a universal family $\mathcal{U}$ exists globally on $\mathbf{M}_{H o d}(X, 1)$.

Proof. We shall give an explicit construction since it will be useful for us in what follows; it would also be possible to modify the proof of the analogous statement for vector bundles with co-prime rank and degree in Newstead [20] to apply to Simpson's GIT construction of the moduli space $\mathbf{M}_{H o d}(X, 1)$ in Simpson [24, 25]. 
By (3.1.5) and (3.2.5), we have a real-analytic isomorphism

$$
\mathbf{M}_{H o d}(X, 1) \cong \mathbf{A}^{1} \times \mathrm{J}(X) \times H^{0}\left(X, \Omega_{X}^{1}\right) .
$$

On $\mathbf{M}_{D o l}(X, 1)$ there is obviously a universal family of Higgs bundles with underlying sheaf $\operatorname{pr}_{\mathrm{J}(X)}^{*} \mathcal{P}$; we wish to expand this to a family over $\mathbf{M}_{H o d}(X, 1)$ using the twistor lines.

At a point $(\xi, \theta) \in \mathrm{J}(X) \times H^{0}\left(X, \Omega_{X}^{1}\right)$, denote the complex structure operator of $\mathcal{L}_{\xi}$ by $\bar{\partial}_{\xi}$. At $(\lambda, \xi, \theta) \in \mathbf{A}^{1} \times \mathrm{J}(X) \times H^{0}\left(X, \Omega_{X}^{1}\right)$ choose as in (3.2.7) the complex structure operator $\bar{\partial}_{\xi}+\lambda \bar{\theta}$ and a $\lambda$-connection $\lambda \partial_{\xi}+\theta$. This gives a real-analytic family $\mathcal{U}$ of rank-1 $\lambda$-connections on $\mathbf{M}_{H o d}(X, 1) \times X$, which clearly restricts to the universal family on $\mathbf{M}_{D o l}(X, 1)$.

The holomorphicity of the family $\mathcal{U}$ in the $\lambda$-direction is clear since $\lambda$ is linear in the defining equations. Due to the trivialisation

$$
\mathbf{M}_{H o d}(X, 1) \times \mathbf{A}^{1} \mathbf{G}_{m} \cong \mathbf{M}_{d R}(X, 1) \times \mathbf{G}_{m}
$$

in (3.2.4), it is enough to check the holomorphicity in the fibre direction for $\mathbf{M}_{d R}(X, 1)$. But it follows from (3.1.5) that the monodromy of $\mathcal{U}$ at

$$
\left(c_{1}, \ldots, c_{2 g}\right) \in \mathbf{M}_{d R}(X, 1)=\left(\mathbf{C}^{*}\right)^{2 g} \cong\left(\mathbf{U}(1) \times \mathbf{R}_{+}^{*}\right)^{2 g}
$$

is $\left(c_{1}, \ldots, c_{2 g}\right)$, and thus the restriction of $\mathcal{U}$ to $\mathbf{M}_{d R}(X, 1) \times X$ is indeed the universal family of flat line bundles.

4. Autodual connection in the Fourier transform. After the technical preliminaries in the previous two sections, we shall now describe the construction of a canonical autodual connection in the Fourier transform $\widehat{E}$ of a Higgs bundle $E$ on a curve $X$. The base space of $\widehat{\mathrm{E}}$ is the hyper-Kähler manifold $\mathbf{M}_{D o l}(X, 1)$, the moduli space of Higgs bundles of degree zero and rank 1. The Fourier transform can be interpreted in the set-up of Section 2 as the first de Rham object of a Simpson module on $\mathbf{M}_{D o l}(X, 1) \times X$ corresponding to the (external) tensor product $\mathrm{E} \otimes \mathcal{U}_{\text {Dol }}$ of $\mathrm{E}$ with the universal Higgs bundle $\mathcal{U}_{\text {Dol }}$.

The first step in the construction of the autodual connection reduces the problem to the construction of a suitable holomorphic vector bundle on the twistor space (Theorem (4.2.7)). Using Deligne's description of the twistor space from the previous section we can then further reduce this to the problem of constructing suitable holomorphic bundles on the "halves" of the twistor space given by $\mathbf{M}_{H o d}(X, 1) \supset \mathbf{M}_{D o l}(X, 1)$. These required bundles can be constructed as the first de Rham sheaves of a natural extension of $\mathrm{E} \otimes \mathcal{U}_{\text {Dol }}$ to a sheaf with $\lambda$-connection on $\mathbf{M}_{H o d}(X, 1) \times X$.

4.1. The Fourier transformation. We recall the construction in [4] of the Fourier transformation for Higgs bundles and provide a translation into the set-up of this paper.

Notation 4.1.1. Let $X$ be a smooth projective curve of genus $g \geq 2$. We denote by $M$ the moduli space $\mathbf{M}_{D o l}(X, 1)$ of rank-1 Higgs bundles on $X$. It is naturally isomorphic to the cotangent bundle of the Jacobian $\mathrm{J}(X)$ of $X$, i.e., to $\mathrm{J}(X) \times H^{0}\left(X, \Omega_{X}^{1}\right)$.

4.1.2. Let $\mathrm{E}=(\mathcal{E}, \theta)$ be a stable Higgs bundle of degree 0 on $X$. By adding to $\theta$ the "Abelian" Higgs fields $\alpha \otimes 1_{\mathcal{E}}$ for $\alpha \in H^{0}\left(X, \Omega_{X}^{1}\right)$, one gets a family $\mathrm{E}(\alpha)$ of Higgs 
bundles parametrised by the affine space $V=H^{0}\left(X, \Omega_{X}^{1}\right)$. We consider this family as a complex of sheaves

$$
\mathcal{H}(\mathrm{E})=\left(\operatorname{pr}_{X}^{*} \mathcal{E} \stackrel{\Theta}{\rightarrow} \operatorname{pr}_{X}^{*}\left(\mathcal{E} \otimes \Omega_{X}^{1}\right)\right)
$$

in degrees 0 and 1 on $X \times V$, and hence as an object of $\mathbf{D}_{c}^{b}(X \times V)$.

Let $\mathcal{M}_{V}$ be the pull-back to $X \times \mathrm{J}(X) \times V=X \times M$ of the universal sheaf $\mathcal{M}$ on $X \times \mathrm{J}(X)$. The Fourier transform of $\mathrm{E}$ is by definition the relative Fourier-Mukai transform

$$
\widehat{\mathrm{E}}=H^{1}\left(\Phi_{X \times V \rightarrow \mathrm{J}(X) \times V / V}^{\mathcal{M}_{V}}(\mathcal{H}(\mathrm{E}))\right)
$$

(see (2.4.4) of [4]). In other words, it is the higher direct image

$$
\widehat{\mathrm{E}}=\mathbf{R}^{1}\left(\operatorname{pr}_{M}\right)_{*}\left(\operatorname{pr}_{X \times V}^{*} \mathcal{H}(\mathrm{E}) \otimes \mathcal{M}_{V}\right) .
$$

We recall that by Proposition (3.1.8) in [4] the Fourier transform of a stable Higgs bundle $\mathrm{E}$ of rank $\geq 2$ and degree 0 is a locally free sheaf.

4.1.3. We translate the above definition into the framework of this paper. The base manifold of $\widehat{\mathrm{E}}$ is the moduli space $M=\mathbf{M}_{D o l}(X, 1)$ of rank-1 Higgs bundles. The complex $\operatorname{pr}_{X \times V}{ }^{*} \mathcal{H}(\mathrm{E}) \otimes \mathcal{M}_{V}$ as an object of $\mathbf{D}_{c}^{b}(X \times M)$ is clearly the "de Rham complex"

$$
\mathbf{D R}_{X \times M / M}\left(\operatorname{pr}_{X}^{*} \mathrm{E} \stackrel{\mathbf{L}}{\otimes} \mathcal{U}\right),
$$

where $\mathcal{U}$ is the universal rank-1 Higgs bundle on $X \times M$ and the tensor product is taken in the derived category of $\operatorname{Sym}^{\bullet}\left(\mathcal{T}_{X \times M / M}\right)$-modules (see section 2.1 ). Thus

$$
\widehat{\mathrm{E}}=\mathbf{R}^{1} \operatorname{pr}_{M *}\left(\mathbf{D R}_{X \times M / M}\left(\operatorname{pr}_{X}^{*} \mathrm{E} \stackrel{\mathbf{L}}{\otimes} \mathcal{U}\right)\right) .
$$

We shall show that this locally free sheaf admits an autodual connection by using a twistorial description reviewed below.

4.2. Autodual connections and twistor transform. We recall the definition of an autodual connection in a bundle on a hyper-Kähler manifold. We then quickly review the results of Kaledin-Verbitsky [16] that provide a characterisation of autodual connections in terms of their twistor transforms, which are holomorphic sheaves on the twistor space. Theorem (4.2.7) then allows us to reduce to the construction of autodual connection to that of a suitable holomorphic vector bundle on the twistor space.

4.2.1. Let $M$ be a hyper-Kähler manifold. The complex structures $I, J$ and $K$ give an action of the quaternions on the tangent bundle $T M$ and hence an action of the group $\mathbf{S U}(2)=\mathbf{S p}(1)$ of unit quaternions. This action extends to tensor and exterior powers, and so in particular to the bundles of differential forms.

Definition 4.2.2. A connection $\nabla$ in a smooth vector bundle $E$ on $M$ is autodual if its curvature 2 -form $F_{\nabla}$ is invariant under the action of $\mathbf{S U}(2)$. 
This generalises the autoduality condition from 4-manifolds to (not necessarily Hermitean) connections on hyper-Kähler manifolds.

4.2.3. Let $E$ be a complex vector bundle on $M$, equipped with a connection $\nabla$. The pull-back $\operatorname{pr}_{M}^{*} E$ on $\mathbf{T w}(M)$ has the natural pull-back connection $\operatorname{pr}_{M}^{*} \nabla$. By Lemma 5.1 of [16] the curvature of $\operatorname{pr}_{M}^{*} \nabla$ is of type $(1,1)$ precisely when $\nabla$ is auto-dual. In particular, for autodual $\nabla$ the pull-back $\operatorname{pr}_{M}^{*} \nabla$ defines a holomorphic structure on $\operatorname{pr}_{M}^{*} E$; the resulting holomorphic bundle on $\mathbf{T w}(M)$ is called the twistor transform of the autodual bundle $(E, \nabla)$. Notice that in particular that the $(0,1)$ part $\nabla^{0,1}$ of the connection defines a holomorphic structure on $E$ with respect to each complex structure on $M$ belonging to the hyper-Kähler family parametrised by $\mathbf{P}^{1}$.

Definition 4.2.4. A holomorphic bundle $\mathcal{F}$ on $\mathbf{T w}(M)$ is called twistorial if the restrictions $\tilde{m}^{*} \mathcal{F}$ are trivial for all horizontal twistor lines $\tilde{m}: \mathbf{P}^{1} \rightarrow \mathbf{T w}(M)$.

Proposition 4.2.5. The twistor transform of an auto-dual connection is twistorial.

Proof. This follows directly from the construction.

4.2.6. Let $\mathcal{F}$ be a twistorial holomorphic bundle on $\mathbf{T w}(M)$ with underlying smooth complex vector bundle $F$ and holomorphic structure operator $\bar{\partial}$. The realanalytic isomorphism $\mathbf{T w}(M) \cong M \times \mathbf{P}^{1}$ induces a splitting

$$
\mathcal{A}^{0,1}(\mathbf{T w}(M))=\operatorname{pr}_{M}^{*} \mathcal{A}^{0,1}(M) \oplus \operatorname{pr}_{\mathbf{P}^{1}}^{*} \mathcal{A}^{0,1}\left(\mathbf{P}^{1}\right)
$$

of the type $(0,1)$ forms. This gives the decomposition

$$
\bar{\partial}=\bar{\partial}_{M}+\bar{\partial}_{\mathbf{P}^{1}}
$$

of $\bar{\partial}: F \rightarrow F \otimes \mathcal{A}^{0,1}$ into operators

$$
\begin{aligned}
& \bar{\partial}_{M}: F \rightarrow F \otimes \operatorname{pr}_{M}^{*} \mathcal{A}_{M}^{0,1}, \\
& \bar{\partial}_{\mathbf{P}^{1}}: F \rightarrow F \otimes \operatorname{pr}_{\mathbf{P}^{1}}^{*} \mathcal{A}_{\mathbf{P}^{1}}^{0,1} .
\end{aligned}
$$

We call smooth sections of $F$ in the kernel $\Gamma_{t w}(\mathbf{T w}(M), F)$ of $\bar{\partial}_{\mathbf{P}^{1}}$ twistor holomorphic sections, and define a twistorial direct image sheaf $\operatorname{pr}_{M+}(\mathcal{F})$ on $M$ by

$$
\Gamma\left(U, \operatorname{pr}_{M+}(\mathcal{F})\right)=\Gamma_{t w}\left(\operatorname{pr}_{M}^{-1}(U), F\right) .
$$

Then $\operatorname{pr}_{M+} \mathcal{F}$ is a sheaf of sections of a smooth complex vector bundle on $M$. Moreover, since $\mathcal{F}$ is twistorial, $\operatorname{pr}_{M}^{*} \operatorname{pr}_{M+} \mathcal{F}=\mathcal{F}$, and the operator $\bar{\partial}_{M}$ gives by adjunction an operator

$$
\nabla: \operatorname{pr}_{M+} \mathcal{F} \rightarrow \operatorname{pr}_{M+}\left(\mathcal{F} \otimes \mathcal{A}_{M}^{0,1}\right)=\operatorname{pr}_{M+} \mathcal{F} \otimes \mathcal{A}^{1}(M)
$$

where the isomorphism results from a version of the projection formula for $\operatorname{pr}_{M+}$. It follows from Lemmas 5.8. and 5.9. of [16] that $\nabla$ is an autodual connection in $\operatorname{pr}_{M+} \mathcal{F}$; the complex vector bundle $\operatorname{pr}_{M+} \mathcal{F}$ with the autodual connection $\nabla$ is called the inverse twistor transform of $\mathcal{F}$.

THEOREM 4.2.7. The twistor transformation and the inverse twistor transformation are quasi-equivalences to each other and establish an equivalence between the categories of bundles with autodual connections on $M$ and holomorphic bundles on $\mathrm{Tw}(M)$ that are trivial along the horizontal twistor lines.

Proof. Theorem 5.12 of Kaledin-Verbitsky [16]. 
4.3. Construction of the twistor transform. We complete the construction of the autodual connection by constructing a suitable twistorial bundle on the twistor space of the base manifold $\mathbf{M}_{D o l}(X, 1)$. The construction of the restrictions of the twistorial bundle to the "halves" of the twistor space isomorphic to the moduli space $\mathbf{M}_{H o d}(X, 1)$ and its conjugate rely on the cohomological machinery developed in Section 2.1. The glueing together of the bundle on the twistor space and the verification that it be twistorial relies on the analytical results of Simpson and Corlette reviewed in Section 3.

Notation 4.3.1. Let $X$ be a smooth comple curve of genus $\geq 2$. We continue to denote by $M$ the hyper-Kähler moduli space $\mathbf{M}_{D o l}(X, 1)$. Let $T=\mathbf{T w}(M)$ be the twistor space. By (3.2.5) it is glued together from $\mathbf{M}_{H o d}(X, 1)$ and $\overline{\mathbf{M}_{H o d}(X, 1)}$ by means of the anti-holomorphic involution $\sigma^{\prime}$ of (3.2.4.2); we denote these "halves" of the twistor space by $T^{+}$and $T^{-}$respectively. Let $\mathcal{U}$ be the universal rank-1 $\lambda$ connection on $T^{+} \times X$, and denote by $\lambda: T^{+} \rightarrow \mathbf{A}^{1}$ the natural fibration.

4.3.2. Let $\mathrm{E}$ be a stable degree- 0 Higgs bundle on $X$. The construction in (3.2.7) of the (half of the) horizontal twistor line through the point in $\mathbf{M}_{D o l}(X, n)$ corresponding to $\mathrm{E}$ gives us a bundle with a $\lambda$-connection $\mathrm{E}^{\prime}$ on $\mathbf{A}^{1} \times X$, which restricts to $\mathrm{E}$ on $\{0\} \times X$. Let

$$
\mathbf{E}^{+}=\left(\lambda \times 1_{X}\right)^{*} \mathbf{E}^{\prime} \stackrel{\mathbf{L}}{\otimes} \mathcal{U}
$$

We consider the object

$$
\mathcal{F}^{+}=\mathbf{R}^{1} \operatorname{pr}_{T^{+*}}\left(\mathbf{D R}_{X \times T^{+} / T^{+}}\left(\mathrm{E}^{+}\right)\right)
$$

of $\mathbf{D}_{c}^{b}\left(T^{+}\right)$. By construction the restriction of $\mathcal{F}^{+}$to $M \subset T^{+}$is the Fourier transform $\hat{\mathrm{E}}$.

Lemma 4.3.3. The sheaf $\mathcal{F}^{+}$constructed above is locally free $\mathcal{O}_{T^{+}}$-module.

Proof. Since $X$ is complete, it follows from (2.1.13) that $\mathcal{F}^{+}$is a coherent $\mathcal{O}_{T^{+-}}$ module. Hence it suffices to show that the dimension of the fibres $\mathcal{F}^{+}(m)$ is constant for $m \in T^{+}=\mathbf{M}_{H o d}(X, 1)$. For $m \in \mathbf{M}_{D o l}(X, 1)$ this follows as the Fourier transform is locally free. But by Corollary 2.3. of Simpson [23]

$$
\mathcal{F}^{+}(m) \cong \mathcal{F}^{+}\left(m^{\prime}\right)
$$

where $m^{\prime} \in \mathbf{M}_{d R}(X, 1)$ corresponds to $m \in \mathbf{M}_{D o l}(X, 1)$ under the homeomorphism of (3.1.4). Finally, for general $m \notin M=\mathbf{M}_{D o l}(X, 1)$ this follows from the trivialisation of $\mathbf{M}_{H o d}(X, 1) \backslash \mathbf{M}_{D o l}(X, 1)$ in (3.2.4) and the fact that multiplication of the differential of a de Rham complex by a constant does not affect its hypercohomology.

4.3.4. Consider the universal Higgs bundle $\left(X \times \mathbf{C}, \bar{\partial}_{\xi}, \theta\right)$ on the base $\mathrm{J}(X) \times$ $H^{0}\left(X, \Omega_{X}^{1}\right) \times X=\mathbf{M}_{D o l}(X, 1) \times X$. Using the harmonic (i.e., product) metric on $L=X \times \mathbf{C}$, we get by (3.1.1) the operators $\bar{\theta}$ and $\partial_{\xi}$. By (3.1.3), $\left(L, \partial_{\xi},-\bar{\theta}_{\xi}\right)$ is a (in fact universal) family of Higgs line bundles on $\overline{\mathbf{M}_{D o l}(X, 1)} \times \bar{X}$. As in the proof of (3.2.8), for each $\lambda \in \mathbf{C}, \partial_{\xi}-\bar{\lambda} \theta_{\xi}$ is a complex structure operator for $L$ on $\bar{X}$, and $\bar{\lambda} \bar{\partial}_{\xi}-\bar{\theta}$ is a $\bar{\lambda}$-connection in $\left(L, \partial_{\xi}-\bar{\lambda} \theta_{\xi}\right)$. It is clear that the family is holomorphic with respect to $T^{-}$. We denote this family of rank-1 $\lambda$-connections on $T^{-} \times \bar{X}$ by $\mathcal{U}^{-}$. 
LEMMA 4.3.5. The anti-holomorphic involution $\tau$ of the moduli space of representations of $\pi_{1}(X)$ used in the glueing of $T$ from $T^{+}$and $T^{-}$exchanges the monodromies of the restrictions of $\mathcal{U}$ and $\mathcal{U}^{-}$to moduli spaces of flat connections.

Proof. First, by 3.1.3, the conjugate family of $\mathcal{U}$ with complex structure operator $\partial_{\xi}+\bar{\lambda} \theta_{\xi}$ and $\bar{\lambda}$-connection $\bar{\lambda} \bar{\partial}_{\xi}+\bar{\theta}$ restricted to $\mathbf{M}_{d R}(\bar{X}, 1)$ has the same monodromy as $\mathcal{U}$.

It follows from (3.1.5.1) that the map $\theta \mapsto-\theta$ corresponds to

$$
\left(\varphi_{1}, \ldots, \varphi_{2 g}, r_{1}, \ldots, r_{2 g}\right) \mapsto\left(\varphi_{1}, \ldots, \varphi_{2 g}, 1 / r_{1}, \ldots, 1 / r_{2 g}\right)
$$

in polar coordinates on $\left(\mathbf{C}^{*}\right)^{2 g}$. But this is just the map

$$
\left(c_{1}, \ldots, c_{2 g}\right) \mapsto\left({\overline{c_{1}}}^{-1}, \ldots,{\overline{c_{2 g}}}^{-1}\right),
$$

i.e., the involution of the moduli space.

4.3.6. Let $E$ and $\bar{\partial}$ denote the underlying smooth complex vector bundle and the holomorphic structure operator of the Higgs bundle E. Using a harmonic metric $h$ on $(E, \bar{\partial}, \theta)$ we get by (3.1.1) and (3.1.2) operators $\bar{\theta}_{h}$ and $\partial_{h}$ such that

$$
\nabla=\partial_{h}+\bar{\partial}+\theta+\bar{\theta}_{h}
$$

is the flat connection corresponding to $E$ by (3.1.2). Now by (3.1.3) the operator $\partial_{h}$ defines in $E$ a structure of a holomorphic bundle on the complex conjugate curve $\bar{X}$, and the operator $-\bar{\theta}_{h}$ makes $\left(E_{h}, \partial_{h}\right)$ into a stable Higgs bundle we denote by $\overline{\mathrm{E}}$.

4.3.7. As in (4.3.2), the Higgs bundle $\bar{E}$ gives us a holomorphic family

$$
\overline{\mathrm{E}}^{\prime}=\left(E, \bar{\lambda} \bar{\partial}-\bar{\theta}_{h}+\partial_{h}-\bar{\lambda} \theta\right)
$$

of $\lambda$-connections on $\bar{X}$ parametrised by $\overline{\mathbf{A}^{1}}$. This is just the family giving a half of the horizontal twistor line corresponding $\overline{\mathrm{E}}$ in the twistor space $T^{-}=\overline{\mathbf{M}_{D o l}(X, 1)}=$ $\mathbf{M}_{\text {Dol }}(\bar{X}, 1)$. Let

$$
\mathrm{E}^{-}=(\lambda \times 1 \bar{X})^{*} \overline{\mathrm{E}}^{\prime} \stackrel{\mathrm{L}}{\otimes} \mathcal{U}^{-}
$$

Consider the object

$$
\mathcal{F}^{-}=\mathbf{R}^{1} \operatorname{pr}_{T^{-*}}\left(\mathbf{D} \mathbf{R}_{X \times T^{-} / T^{-}} \mathrm{E}^{-}\right) .
$$

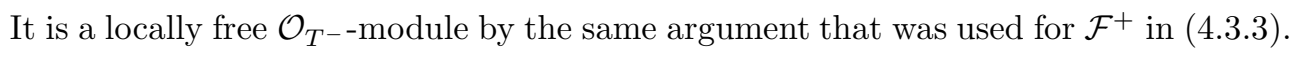

Proposition 4.3.8. Let $\sigma^{\prime}: T^{+} \times{ }_{\mathbf{A}^{1}} \mathbf{G}_{m} \rightarrow T^{-} \times \overline{\mathbf{A}^{1}} \overline{\mathbf{G}_{m}}$ be the morphism used to glue together the twistor space $T$ in (3.2.4). Then the pulled-back vector bundle $\sigma^{\prime *}\left(\mathcal{F}^{-}\right)$is isomorphic to the restriction of $\mathcal{F}^{+}$on $T^{+} \times{ }_{\mathbf{A}^{1}} \mathbf{G}_{m}$.

Proof. Consider the morphism

$$
f=\left(\sigma^{\prime} \times 1_{X}\right): T^{+} \times \mathbf{A}^{1} \mathbf{G}_{m} \times X \rightarrow T^{-} \times \overline{\mathbf{A}^{1}} \overline{\mathbf{G}_{m}} \times \bar{X} .
$$

Notice that $f$ is a morphism of schemes, but not a morphism of $\mathbf{C}$-schemes. Since $\mathcal{F}^{-}=\mathbf{R}^{1} \operatorname{pr}_{T^{-*}}\left(\mathrm{E}^{-}\right)$is locally free, it follows by base change that

$$
\sigma^{\prime *}\left(\mathcal{F}^{-}\right)=\mathbf{R}^{1} \operatorname{pr}_{T^{+*}}\left(f^{*}\left(\mathrm{E}^{-}\right)\right) .
$$


Denote $N=\mathbf{M}_{d R}(X, 1)$. Let $\mathrm{E}_{1}^{+}$be the restriction of $\mathrm{E}^{+}$to $N \times X$, and let $\mathcal{F}_{1}^{+}=\mathbf{R}^{1} \mathrm{pr}_{N *} \mathrm{E}_{1}^{+}$be the restriction of $\mathcal{F}^{+}$to $N \subset T^{+}$. Now on $T^{+} \times{ }_{\mathbf{A}^{1}} \mathbf{G}_{m}=N \times \mathbf{G}_{m}$ we have the family $\lambda^{-1} \mathrm{E}^{+}$of flat connections by (3.2.4). But since multiplication of the differentials in a complex does not affect its hypercohomology, it follows that the restriction of $\mathcal{F}^{+}$to $N \times \mathbf{G}_{m}$ is the pull-back $\operatorname{pr}_{N}^{*} \mathcal{F}_{1}^{+}$. Similarly, let $\mathrm{E}_{1}^{-}$denote the restriction of $\mathrm{E}^{-}$to $\mathbf{M}_{d R}(\bar{X}, 1) \times \bar{X}$; then we see that the restriction of $\mathcal{F}^{-}$to $T^{-} \times \overline{\mathbf{A}^{1}} \times \overline{\mathbf{G}_{m}}=\bar{N} \times \overline{\mathbf{G}_{m}}$ is the pull-back of $\mathcal{F}_{1}^{-}=\mathbf{R}^{1} \mathrm{pr}_{\bar{N} *} \mathrm{E}_{1}^{-}$.

Hence it is enough to find an isomorphism

$$
\mathbf{R}^{1} \operatorname{pr}_{N *}\left(\mathrm{E}_{1}^{+}\right) \stackrel{\sim}{\rightarrow} \mathbf{R}^{1} \operatorname{pr}_{N *}\left(f^{*} \mathrm{E}_{1}^{-}\right)
$$

But now the proposition follows from the following lemma.

Lemma 4.3.8.1. There is an isomorphism $f^{*}\left(\mathrm{E}^{-}\right) \rightarrow \mathrm{E}^{+}$on $T^{+} \times \mathbf{A}^{1}\{1\}=$ $\mathbf{M}_{d R}(X, 1)$.

Let $\mathcal{L}^{+}$be the family of local systems (i.e., locally constant sheaves) on $N \times X$ for which $\mathrm{E}_{1}^{+}=\mathcal{L}^{+} \otimes_{\mathbf{C}} \mathcal{O}_{N \times X}$, and let $\mathcal{L}^{-}$be the family of local systems on $\bar{N} \times \bar{X}$ for which $\mathrm{E}_{1}^{-}=\mathcal{L}^{-} \otimes_{\mathbf{C}} \mathcal{O}_{\bar{N} \times \bar{X}}$. Then $f^{*} \mathrm{E}_{1}^{-}=f^{-1} \mathcal{L}^{-} \otimes_{\mathbf{C}} \mathcal{O}_{N \times X}$. But it follows from (3.1.3), (3.2.6) and (4.3.5) that $f^{-1} \mathcal{L}^{-} \cong \mathcal{L}^{+}$, whence the lemma.

4.3.9. The proposition allows us to glue the sheaves $\mathcal{F}^{+}$on $T^{+}$and $\mathcal{F}^{-}$on $T^{-}$ together into a sheaf on $T$. Let us denote this sheaf by $\mathcal{F}$. Notice that the glueing map is essentially constant in the $\mathbf{G}_{m}$-direction, being pulled back from the glueing map for $\mathbf{M}_{d R}(X, 1) \subset T$.

LEMma 4.3.10. The glued-together sheaf $\mathcal{F}$ constructed in (4.3.9) is a twistorial locally free $\mathcal{O}_{T}$-module.

Proof. That $\mathcal{F}$ is locally free is clear since it is glued from two locally free sheaves. We need to show that it is twistorial. Let $\tilde{m}: \mathbf{P}^{1} \rightarrow T$ be a horizontal twistor line. Then formality Lemma 2.2 in Simpson [23] gives trivialisations of $\tilde{m}^{*} \mathcal{F}$ over $\mathbf{A}^{1}$ and $\overline{\mathbf{A}^{1}}$. Since the glueing in (4.3.9) is propagated from the isomorphism $\tilde{m}^{*} \mathcal{F}(1) \rightarrow$ $\tilde{m}^{*} \mathcal{F}(-1)$, the transition function will be constant. Hence the locally free sheaf $\tilde{m}^{*} \mathcal{F}$ is in fact globally free.

Proposition 4.3.11. The underlying vector bundle of the inverse twistor transform of $\mathcal{F}$ is $\widehat{\mathrm{E}}$.

Proof. Let $(E, \nabla)$ be the inverse twistor transform of $\mathcal{F}$. Then by (4.2.7) $\mathcal{F}$ is the twistor transform of $(E, \nabla)$, i.e., $\operatorname{pr}_{M}^{*} E$ with the complex structure given by the $(0,1)$-part of the pull-back connection $\operatorname{pr}_{M}^{*} \nabla$. But it is clear that the underlying smooth bundle of $\widehat{E}=\left.\mathcal{F}\right|_{M}$ is $E$.

THEOREM 4.3.12. The Fourier transform $\widehat{\mathrm{E}}$ of a stable Higgs bundle $\mathrm{E}$ of rank $\geq 2$ and degree 0 on a smooth complete curve $X$ of genus $\geq 2$ has a natural autodual connection.

Proof. This follows from (4.3.10), (4.2.7) and (4.3.11).

REMARKS 4.3.13.

1. A second, alternative, construction of the Fourier transform and the autodual connection for stable Higgs bundles on curves was outlined in [3, page 66]. That approach, originally suggested by Nigel Hitchin (see the discussion in 
Hitchin [13, Section 7]), represents the Fourier transform as the bundle of kernels of a suitable family of Dirac operators coupled to the Higgs field, modelled on the Nahm transform for monopoles. This was also effectively the approach taken in Jardim [15] for the case of singular Higgs bundles on genus-1 curves. This alternative construction has been recently carried out for stable Higgs bundles on curves of genus $\geq 2$ by Frejlich-Jardim in [10]. They obtain analytic proofs for many of the results in [4] (specialised to the setting of complex geometry) as well as the existence of the autodual connection, thereby confirming the expectations stated in [3].

2. Related questions have been studied in a few other recent articles. A Nahm transform for singular Higgs bundles on curves of genus 0 is defined by an analytic approach in Szabó [27]. Tejero-Prieto [28] relates the general Fourier transformation for curves defined in [4] (used to construct the Fourier transformation for Higgs bundles) to a corresponding Nahm-type transformation, and applies these to study certain spectral bundles on the Jacobian. This effectively describes related Fourier and Nahm transforms for stable bundles (the case of vanishing Higgs field). In addition, in [1] Bartocci and Biswas described a different type of Fourier-Mukai transform for Higgs bundles over varieties of arbitrary dimension.

\section{REFERENCES}

[1] C. Bartocci and I. Biswas, Higgs bundles and the Fourier-Mukai transform, Southeast Asian Bull. Math., 25:2 (2001), pp. 201-207.

[2] J.-E. BJöRK, Analytic D-modules and applications, Kluwer, Dordrecht, 1993.

[3] J. BonsdorfF, A Fourier Transform for Higgs Bundles, PhD thesis, University of Oxford, 2002.

[4] J. BonsdorfF, A Fourier transformation for Higgs bundles, J. Reine Angew. Math., 591 (2006), pp. 21-48.

[5] A. Borel ET AL, Algebraic D-modules, Academic Press, Boston, MA, 1987.

[6] M. Mamone Capria and S. M. Salamon, Yang-Mills fields on quaternionic spaces, Nonlinearity, 1:4 (1988), pp. 517-530.

[7] K. Conlette, Flat G-bundles with canonical metrics, J. Differential Geom., 28:3 (1988), pp. 361-382.

[8] P. Deligne, Letter to Simpson, unpublished, March 1989.

[9] S. K. Donaldson, Twisted harmonic maps and the self-duality equations, Proc. London Math. Soc. (3), 55:1 (1987), pp. 127-131.

[10] P. Frejlich and M. Jardim, Nahm transform for Higgs bundles, J. Geom. Phys., 58:9 (2008), pp. $1221-1230$.

[11] A. FuJIKI, Hyper-Kähler structure on the moduli space of flat bundles, in "Prospects in complex geometry (Katata and Kyoto, 1989)", pp. 1-83, Springer, Berlin, 1991.

[12] N. J. Hitchin, Self-duality equations on a Riemann surface, Proc. London Math. Soc. (3), 55 (1987), pp. 59-126.

[13] N. J. Hitchin, The Dirac operator, in Martin Bridson and Simon Salamon, editors, "Invitations to Geometry and Topology", pp. 208-232. Oxford University Press, Oxford, 2002.

[14] N. J. Hitchin, A. Karlhede, U. Lindstrom, And M. RoceK, Hyper-Kahler metrics and supersymmetry, Comm. Math. Phys., 108:4 (1987), pp. 535-589.

[15] M. Jardim, Construction of doubly-periodic instantons, Comm. Math. Phys., 216:1 (2001), pp. $1-15$.

[16] D. Kaledin and M. Verbitsky, Non-Hermitian Yang-Mills connections, Sel. Math., New Ser., 4:2 (1998), pp. 279-320.

[17] G. Laumon, Transformation de Fourier généralisée, preprint, alg-geom/9603004, 1996.

[18] B. Mazur and W. Messing, Universal Extensions and One Dimensional Crystalline Cohomology, Lecture Notes in Mathematics 370, Springer-Verlag, Heidelberg, 1974.

[19] Z. Mebkhout, Le formalisme des six opérations de Grothendieck pour les $\mathcal{D}_{X}$-modules cohérents, Hermann, Paris, 1989. 
[20] P. E. Newstead, Introduction to moduli problems and orbit spaces, Tata Institute of Fundamental Research, Bombay, 1978.

[21] S. Salamon, Quaternionic Kähler manifolds, Invent. Math., 67:1 (1982), pp. 143-171.

[22] C. T. Simpson, Constructing variations of Hodge structure using Yang-Mills theory and applications to uniformization, J. Amer. Math. Soc., 1:4 (1988), pp. 867-918.

[23] C. T. Simpson, Higgs bundles and local systems, Inst. Hautes Études Sci. Publ. Math., 75 (1992), pp. 5-95.

[24] C. T. Simpson, Moduli of representations of the fundamental group of a smooth projective variety. I, Inst. Hautes Études Sci. Publ. Math., 79 (1994), pp. 47-129.

[25] C. T. Simpson, Moduli of representations of the fundamental group of a smooth projective variety. II, Inst. Hautes Études Sci. Publ. Math., 80 (1994), pp. 5-79.

[26] C. T. Simpson, The Hodge filtration on nonabelian cohomology, in "Algebraic geometry-Santa Cruz 1995", pp. 217-281, Amer. Math. Soc., Providence, RI, 1997.

[27] S. Szabó, Nahm transform for integrable connections on the Riemann sphere, Mém. Soc. Math. Fr., Nouv. Sér., 110 (2007), pp. 1-114.

[28] C. Tejero-Prieto, Fourier-Mukai transform and adiabatic curvature of spectral bundles for Landau Hamiltonians on Riemann surfaces, Comm. Math. Phys., 265:2 (2006), pp. 373396.

[29] M. VeRBitsky, Deformations of trianalytic subvarieties of hyperkähler manifolds, Sel. Math., New Ser., 4:3 (1998), pp. 447-490. 
J. BONSDORFF 